\title{
No lockdown for neurological diseases during COVID19 pandemic infection
}

\author{
Valeria Caso $^{1} \cdot$ Antonio Federico $^{2}$ \\ Published online: 8 April 2020 \\ (C) Fondazione Società Italiana di Neurologia 2020
}

Twenty-twenty will be remembered as the year that brought new and unprecedented challenges to the healthcare system worldwide due to the Covid-19 pandemic. Currently, onethird of the world population is living in a coronavirus "lockdown modus" as part of the world's most massive and restrictive mass quarantine.

The first epidemic of cases with unexplained low respiratory infections detected in Wuhan, the largest metropolitan area in China's Hubei province, was first reported to the WHO Country Office in China on December 31st, 2019 [1] In a meeting on January 30th, 2020, per the International Health Regulations (IHR, 2005), the outbreak was declared by the WHO a Public Health Emergency of International Concern (PHEIC) as it had spread to 18 countries with four countries reporting human-to-human transmission. WHO Director-General's statement on the IHR Emergency Committee on Novel Coronavirus (2019-nCoV) [2].

Subsequently, the task of experts of the International Committee on Taxonomy of Viruses (ICTV) termed it the SARS-CoV-2 virus as it is very similar to the one that caused the SARS outbreak (SARS-CoVs). On February 11th, 2020, the WHO Director-General, Dr. Tedros Adhanom Ghebreyesus, announced that the disease caused by this new CoV was a "COVID-19," which is the acronym of "coronavirus disease 2019" [2].

The potential for these viruses to grow and to become a pandemic worldwide seems to be a serious public health risk. Concerning COVID-19, the WHO raised the threat to the CoV epidemic to the "very high" level, on February 28th, 2020. On

Antonio Federico

antonio.federico@unisi.it

1 Cerebrovascular Diseases, Stroke Unit, Perugia Hospital, Perugia, Italy

2 Neurological Sciences, Department, Medicine, Surgery and Neurosciences, University of Siena, Viale Bracci 2, 53100 Siena, Italy
March 11th, as the number of COVID-19 cases outside China has increased 13 times and the number of countries involved has tripled with more than 118,000 cases in 114 countries, and over 4000 deaths, WHO declared the COVID-19 a pandemic [3].

Since the first case diagnosed on February 20th, 2020, in the Codogno Hospital (Lodi, Lombardy, Italy) [4], the infection has rapidly spread throughout Lombardy and the peninsula, reaching 97.689 confirmed cases, with 10.779 deaths as of March 29th, 2020. In Italy, in geographic regions of the north, initially, and subsequently throughout the country, political and health authorities are making incredible efforts to contain a shock wave that is severely testing the health system [5]. Therefore, the attention of healthcare providers is primarily focused on infected patients and the frontline responders.

Nonetheless, the healthcare system needs to continue to respond to the needs of patients affected by noncommunicable diseases [6], which are the majority of conditions. In this regard, neurological disorders contribute to $11,6 \%$ of global DALYs and $16,5 \%$ of deaths from all causes, remaining the leading group cause of DALYs and the second leading group cause of deaths in the world [7]. Preparedness to continue to guarantee treatment to neurological patients is vital in containing and mitigating the collateral effects of COVID-19, which, based on current knowledge, does not seem to be a neurotropic virus and is reported to cause limited neurologic involvements [8].

Baracchini et al., in this brief report published in this current issue, are sharing how the University Hospital of Padua, with a catchment area of about 950.000 people, structured a pathway for acute stroke patients before admitting them to our Stroke Unit [9]. Since the outbreak of the COVID epidemic, a dedicated stroke triage protocol has been activated. The stroke patient who is already known to be COVID-19 positive undergoes Cerebral CT, CTA, and CTP on a high-performance mobile CT outside the Emergency Department. In the case of ischemic stroke without large vessel occlusion, the patient is transferred to the Infectious Disease Department or a dedicated Medical Department after thrombolysis treatment and will 
be continued to be clinically monitored by the stroke team and by the internist. In case of large vessel occlusion, the patient is admitted to the Angio-suite of the Neuroradiology Unit for endovascular treatment, and the personnel is pre-alerted for adopting adequate PPEs. At the end of the procedure, the patient is transferred to the Infectious Disease ward or a dedicated Intensive Care Unit (ICU) dedicated to COVID-19 patients based on neurological conditions.

When the patient is admitted with clinical suspicion of COVID-19 infection because of current fever or recent fever and/or flu-like syndrome, nasopharyngeal swabs are taken from the nasopharynx and each nostril. In the meanwhile, the patient undergoes Cerebral CT, CTA, and CTP on the mobile CT unit. Patients deemed eligible for intravenous thrombolysis follow the standard protocol. If there is no indication for thrombectomy, the patient waits for the result of the COVID-19 screening in a dedicated area, where the stroke team clinically monitors him. Only if the COVID-19 screening is negative, the patient is transferred to the Stroke Unit; whereas the COVID-19 positive patients will be transferred to a dedicated COVID-19 medical ward. In case of large vessel occlusion, the patient is transferred to the Angio suite of the Neuroradiology Unit for endovascular treatment, and the personnel is pre-alerted as for COVID positive. If the COVID-19 screen is negative, the patient is transferred to the Stroke Unit or the Neuro-ICU based on their neurological conditions.

In all cases, the stroke team is responsible for the stroke care of the patient.

The advantages of these pathways are 1) protect the Stroke Unit and personnel from COVID-19 infection, 2) maintain continuous open access to all stroke patients, 3) guarantee to COVID positive patients access to specialized care.

However, we think that a total protection cannot be guaranteed with COVID-19 screen negative patients, because this screen may be negative if done earlier after the infection.

Despite all these efforts, compared to the same period in 2019 , the authors observed a reduction of stroke admission and transfers from spoke centers; specifically, this reduction was $50 \%$ for minor stroke and TIA. Patients who are not admitted to a dedicated stroke unit where etiological diagnostic workup is performed to prescribe tailored secondary prevention treatment will experience a higher rate of stroke recurrences and worse outcomes [10].

Moreover, Baracchini et al. reported that patients tend to arrive too late to be eligible for acute treatment, and the thrombolysis rate and combined thrombolysis and thrombectomy rate reduced by $-26 \%$ and $-30 \%$, respectively. Notably, the primary thrombectomy increased by $41 \%$, which was probably due to the delay in transportation to the hospital.

A possible solution to increase thrombolysis treatment and a correct selection of patients to be transferred to tertiary hospitals is a telestroke-network, allowing patients to be treated in peripheral hospitals and avoiding futile transports [11].
As the Authors correctly stated, the general population, family doctors, and emergency services are currently focused on COVID-19, and they tend to avoid hospital admission for elderly, hypertensive, diabetic, obese, cardiopathic patients because they have a higher mortality rate in the case of COVID-19 infection. In fact, on March 20th, the ISS reported the analysis of the first 3200 deceased patients. The mean age was 78,5 (median: 80, Range 31-103, Range InterQuartile IQR 73-85), whereas 30\% had ischemic heart disease, $22 \%$ AF, 73,8 Hypertension, and 33\% Diabetes Mellitus. These risk profiles are the same as those who are at high risk for stroke [12].

Therefore, protecting patients and keeping "clean" the Stroke Unit environment is crucial because Stroke Unit Care reduces mortality and disability [13].

Another brief communication published in this issue by Bonavita et al., from the Campania Luigi Vanvitelli University, Naples describes a digital triage for patients with Multiple Sclerosis (MS) COVID-19 pandemic [14].

MS, together with stroke and neurodegenerative diseases, is one of the most frequent neurologic disorders. MS is the unique disorder that is only managed by neurologists, while in the other neurological conditions, geriatrics, internal medicine specialists, etc. are frequently involved.

During the COVID-19 pandemic, these patients, being on immunotherapy and belonging - due to their disability and/or comorbidities - to the vulnerable proportion of the population need special attention to minimize their risk of infection.

Based on the experience by the Mount Sinai Hospital in New York to survey immunosuppressed patients with inflammatory bowel disease, the authors developed a digital triage tool to be shared with the MS patients based on Google Forms (https://docs.google.com/forms/u/0). This tool can be easily used by patients to collect the answers and provide a continuity of care to all patients.

The use of telemedicine in neurology has been reported in many recent articles in the care of different neurologic conditions [15-20] and neurorehabilitation [21, 22]. Several articles on this issue have also been published in our journal [23, 24].

With the COVID19 world pandemic infection, the development and applications of these techniques are urgent and will be probably speeded up.

The American Academy of Neurology prepared a document named "Telemedicine and Remote Care Implementation Guide" (American Academy of Neurology web seminar on telemedicine and COVID19 infections (https://www.youtube.com/watch?v=Pw-Jdy3T9g) [25], and an interesting article, under publication and now online, that discusses the most common forms of non-face-to-face care and the proper coding elements necessary to provide these service [15]. 


\section{Conclusions}

We are sure that COVID19 pandemic infection will give us, as neurologists, an excellent opportunity to think about our better care organization for all neurologic disorders, and as scientists to have the possibility of faster exchange of data and results with all the international community. This will prevent a lockdown for all our neurological patients and give them a safe approach to cures in the hospital or at home.

\section{Compliance with ethical standards}

Conflict of interest The authors declare they have no conflict of interest.

Ethical approval None

\section{References}

1. Chan JF-W, Yuan S, Kok K-H et al (2020) A familial cluster of pneumonia associated with the 2019 novel coronavirus indicating person-to-person transmission: a study of a family cluster. Lancet 395:514-523

2. Cascella M, Rajnik M, Cuomo A, et al. (2020). Features, Evaluation and Treatment Coronavirus (COVID-19) [Updated 2020 Mar 20]. In: StatPearls [internet]. Treasure Island (FL): StatPearls Publishing. Available from: https://www.ncbi.nlm.nih. gov/books/NBK554776/

3. https://www.who.int/dg/speeches/detail/who-director-general-sopening-remarks-at-the-media-briefing-on-covid-19\%2D\%2D-11march-2020

4. Grasselli G, Pesenti A, Cecconi M. (2020). Critical care utilization for the COVID-19 outbreak in Lombardy, Italy: early experience and forecast during an emergency response. JAMA. Published online march 13. doi:https://doi.org/10.1001/jama.2020.4031

5. Remuzzi A, Remuzzi G (2020) COVID-19 and Italy: what next? [published online ahead of print, 2020 mar 13]. Lancet S01406736(20):30627-30629. https://doi.org/10.1016/S0140-6736(20) 30627-9)

6. Martinez R, Lloyd-Sherlock P, Soliz P, Ebrahim S, Vega E, Ordunez P, McKee M (2020) Trends in premature avertable mortality from non-communicable diseases for 195 countries and territories, 1990-2017: a population-based study. Lancet Glob Health 8(4):e511-e523. https://doi.org/10.1016/S2214-109X(20)30035-

7. GBD 2016 Neurology Collaborators (2019) Global, regional, and national burden of neurological disorders, 1990-2016: A systematic analysis for the global burden of disease study 2016. Lancet Neurol 18(5):459-480

8. Mao L, Wang W, Chen S, et al (2020). Neurological Manifestations of Hospitalized Patients with COVID-19 in Wuhan, China: a retrospective case series study. The Lancet Neurology, submitted, on line without pre-review. doi: https://doi.org/10.1101/2020.02.22. 20026500

9. Baracchini C. Pieroni A, Viaro F et al (2020) Acute stroke management pathway during Coronavirus- 19 pandemic Neurological Sciences, this issue
10. Lavallée PC, Meseguer E, Abboud H et al (2007) A transient ischaemic attack clinic with round-the-clock access (SOS-TIA): feasibility and effects. Lancet Neurol 6(11):953-960. https://doi.org/10. 1016/S1474-4422(07)70248-X)

11. Hubert GJ, Santo G, Vanhooren G, Zvan B, Tur Campos S, Alasheev A, Abilleira S, Corea F (2019) Recommendations on telestroke in Europe. Eur Stroke J 4(2):101-109. https://doi.org/ $10.1177 / 2396987318806718$

12. https://www.epicentro.iss.it/coronavirus/bollettino/Report-COVID201926 marzo eng.pdf

13. Warlow $\bar{C}$, Sudlow C, Dennis M et al (2003) Stroke. Lancet 362: 1211-1224

14. Bonavita S. Tedeschi G, Atreja A, Lavorgna L. (2020). Digital triage for people with multiple sclerosis in the age of COVID-19 pandemic infection. Neurol. Sci. This issue

15. Cohen BH, Busis NA, Ciccarelli L. (2020) Coding in the world of COVID-19: non-face-to-face evaluation and management. Continuum: Lifelong Learn Neurol-Neurol Syst Dis, 26, (3)

16. Gabriel KMA, Jírů-Hillmann S, Kraft P, Selig U, Rücker V, Mühler J, Dötter K, Keidel M, Soda H, Rascher A, Schneider R, Pfau M, Hoffmann R, Stenzel J, Benghebrid M, Goebel T, Doerck S, Kramer D, Haeusler KG, Volkmann J, Heuschmann PU, Fluri F (2020) Two years' experience of implementing a comprehensive telemedical stroke network comprising in mainly rural region: the Transregional Network for Stroke Intervention with Telemedicine (TRANSIT-Stroke). BMC Neurol 20(1):104

17. Guzik AK, Switzer JA (2020) Teleneurology is neurology. Neurology. 94(1):16-17

18. Hatcher-Martin JM, Adams JL, Anderson ER et al (2020) Telemedicine in neurology: Telemedicine Work Group of the American Academy of Neurology update. Neurology 94(1):30-38

19. Srinivasan R, Ben-Pazi H, Dekker M, et al. (2020) Telemedicine for Hyperkinetic Movement Disorders. Tremor other Hyperkinet Mov (N Y). 10. doi: https://doi.org/10.7916/tohm.v0.698

20. Wilson AM, Jamal NI, Cheng EM, Inkelas M, Saliba D, Hanssen A, Torres JA, Ong MK (2020) Teleneurology clinics for polyneuropathy: a pilot study. J Neurol 267(2):479-490

21. Lei C, Sunzi K, Dai F, Liu X, Wang Y, Zhang B, He L, Ju M (2019) Effects of virtual reality rehabilitation training on gait and balance in patients with Parkinson's disease: A systematic review. PLoS One 14(11):e0224819

22. Torrisi M, Maresca G, De Cola MC et al (2019) Using telerehabilitation to improve cognitive function in post-stroke survivors: is this the time for the continuity of care? Int J Rehabil Res 42(4):344-351

23. Cai G, Huang Y, Luo S, Lin Z, Dai H, Ye Q (2017) Continuous quantitative monitoring of physical activity in Parkinson's disease patients by using wearable devices: a case-control study. Neurol Sci 38(9):1657-1663

24. Nardetto L, Dario C, Tonello S, Brunelli MC, Lisiero M, Carraro MG, Saccavini C, Scannapieco G, Giometto B (2016) A one-to-one telestroke network: the first Italian study of a web-based telemedicine system for thrombolysis delivery and patient monitoring. Neurol Sci 37(5):725-730

25. American Academy of Neurology web seminar on telemedicine and COVID19 infections (https://www.youtube.com/watch?v= Pw-Jdy3-T9g2

Publisher's note Springer Nature remains neutral with regard to jurisdictional claims in published maps and institutional affiliations. 